\title{
A DISCUSSION ON THE STIFFNESS MATRICES USED IN TENSEGRITY STRUCTURES
}

\author{
Luisa María Gil-Martín* \\ University of Granada (UGR), Granada, Spain \\ Manuel Alejandro Fernandez-Ruiz \\ University of Granada (UGR), Granada, Spain \\ Enrique Hernandez-Montes \\ University of Granada (UGR), Granada, Spain
}

The expression stiffness matrices used in the study of tensegrity structures has inherited a formulation initially conceived for the shape finding problems of tension structures. Nevertheless stiffness and geometrical stiffness matrices are well known concepts of structural analysis and all the formulations which are used need to be congruent. In this paper, we present a formal discussion about the formulations used in several publications showing that they are not equivalent. A classic example is presented, which has been solved using several expressions available in the relevant literature, the results have been compared with a classical finite element software, showing the discrepancies.

Key words: Matrices, Stiffness, Structure, US

\section{INTRODUCTION}

Stiffnessis a concept that has been widely studied in the context of tensegrity structures. Since a tensegrity structure is a pin-jointed structure, the classic formulation of structural analysis has to be applicable [01]. For a general understanding, Force=Stiffness $\bullet$ displacement.

In structural analysis, once the stiffness matrix of each element in global coordinates has been obtained (using the transformation matrix, TFigure 1-), the structural stiffness matrix of the whole structure, $\mathrm{KT}$, is obtained by appropriately assembling the stiffness matrices of each one of its members.
For the sake of simplicity and without loss of generality, just 2-D elementsare going to be considered in this work.

A 2-D truss element is represented in Figure 1. It goes from node 1 to node 2 . The Cartesian coordinates of these nodes with respect to the global reference system are (x1, y1) and $(x 2, y 2)$ respectively. In Figure 1 the transformation matrix, T, which connects local and global axes is indicated. We have been using this uncommon order of coordinates to connect it with the notation used in the shape-finding problem of tension-compression structures.

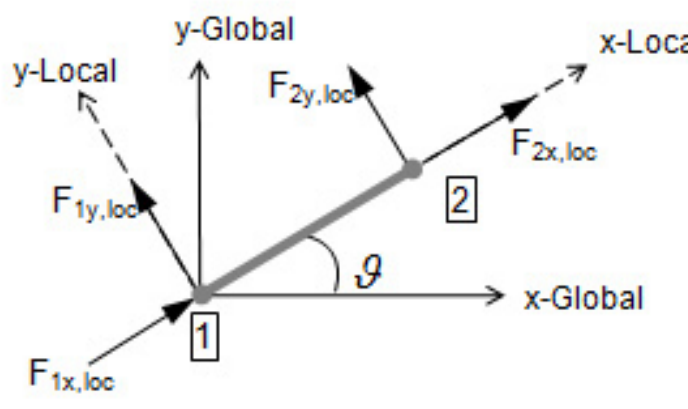

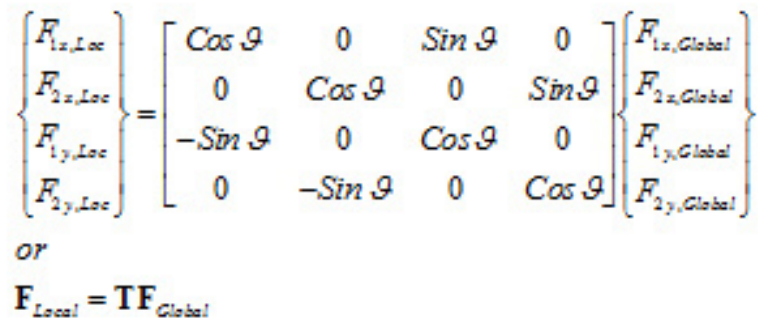

Figure 1: Transformation matrix for a 2-d truss. 
From structural analysis, it is known that the linear stiffness matrix and the geometrical stiffness matrix of a 2-D truss member in local coordinates are given respectively, by:

$$
\mathbf{K}_{E, \text { Local }}=\frac{E \Omega}{L}\left(\begin{array}{cccc}
1 & -1 & 0 & 0 \\
-1 & 1 & 0 & 0 \\
0 & 0 & 0 & 0 \\
0 & 0 & 0 & 0
\end{array}\right)
$$

and $\quad \mathbf{K}_{G, L o c a l}=\frac{N}{L}\left(\begin{array}{cccc}0 & 0 & 0 & 0 \\ 0 & 0 & 0 & 0 \\ 0 & 0 & 1 & -1 \\ 0 & 0 & -1 & 1\end{array}\right)$

with $\mathrm{N}$ as the axial load acting in the member.

Using the transformation matrix, $\mathrm{T}$, given in Figure 1, the local stiffness matrix indicated in Eq. (1) can be expressed in global coordinates as:

$$
\mathbf{K}_{\text {EGbabal }}=\mathbf{T}^{T} \mathbf{K}_{E, \text { Local }} \mathbf{T} \text { and } \mathbf{K}_{G, G b a b l}=\mathbf{T}^{T} \mathbf{K}_{G, L a x a l} \mathbf{T}
$$

Operating in Eq. (2) the following values of both matrices of the member in Figure 1 have been obtained:

$$
\begin{array}{r}
\mathbf{K}_{\mathrm{r}, \mathrm{Glob} \text { al }}=\frac{E \Omega}{L}\left(\begin{array}{cccc}
\operatorname{Cos}^{2} \vartheta & -\operatorname{Cos}^{2} \vartheta & \operatorname{Cos} \vartheta \operatorname{Sin} \vartheta & -\operatorname{Cos} \vartheta \operatorname{Sin} \vartheta \\
-\operatorname{Cos}^{2} \vartheta & \operatorname{Cos}^{2} \vartheta & -\operatorname{Cos} \vartheta \operatorname{Sin} \vartheta & \operatorname{Cos} \vartheta \operatorname{Sin} \vartheta \\
\operatorname{Cos} \vartheta \operatorname{Sin} \vartheta & -\operatorname{Cos} \vartheta \operatorname{Sin} \vartheta & \operatorname{Sin}^{2} \vartheta & -\operatorname{Sin}^{2} \vartheta \\
-\operatorname{Cos} \vartheta \operatorname{Sin} \vartheta & \operatorname{Cos} \vartheta \operatorname{Sin} \vartheta & -\operatorname{Sin}^{2} \vartheta & \operatorname{Sin}^{2} \vartheta
\end{array}\right) \\
\mathbf{K}_{\mathrm{G}, \mathrm{Clob}}=\frac{N}{L}\left(\begin{array}{cccc}
\operatorname{Sin}^{2} \vartheta & -\operatorname{Sin}^{2} \vartheta & -\operatorname{Cos} \vartheta \operatorname{Sin} \vartheta & \operatorname{Cos} \vartheta \operatorname{Sin} \vartheta \\
-\operatorname{Sin}^{2} \vartheta & \operatorname{Sin}^{2} \vartheta & \operatorname{Cos} \vartheta \operatorname{Sin} \vartheta & -\operatorname{Cos} \vartheta \operatorname{Sin} \vartheta \\
-\operatorname{Cos} \vartheta \operatorname{Sin} \vartheta & \operatorname{Cos} \vartheta \operatorname{Sin} \vartheta & \operatorname{Cos}^{2} \vartheta & -\operatorname{Cos}^{2} \vartheta \\
\operatorname{Cos} \vartheta \operatorname{Sin} \vartheta & -\operatorname{Cos} \vartheta \operatorname{Sin} \vartheta & -\operatorname{Cos}^{2} \vartheta & \operatorname{Cos}^{2} \vartheta
\end{array}\right)
\end{array}
$$

From Figure 1 it is evident that:

$$
\begin{aligned}
& \operatorname{Cos} \vartheta=\frac{x_{2}-x_{1}}{r} \text { and } \operatorname{Sin} \vartheta=\frac{y_{2}-y_{1}}{r} \\
& \mathbf{K}_{z, G \text { G bat }}=\frac{E \Omega}{L^{3}}\left(\begin{array}{cccc}
\left(x_{1}-x_{2}\right)^{2} & -\left(x_{1}-x_{2}\right)^{2} & \left(x_{1}-x_{2}\right)\left(y_{1}-y_{2}\right) & -\left(x_{1}-x_{2}\right)\left(y_{1}-y_{2}\right) \\
-\left(x_{1}-x_{2}\right)^{2} & \left(x_{1}-x_{2}\right)^{2} & -\left(x_{1}-x_{2}\right)\left(y_{1}-y_{2}\right) & \left(x_{1}-x_{2}\right)\left(y_{1}-y_{2}\right) \\
\left(x_{1}-x_{2}\right)\left(y_{1}-y_{2}\right) & -\left(x_{1}-x_{2}\right)\left(y_{1}-y_{2}\right) & \left(y_{1}-y_{2}\right)^{2} & -\left(y_{1}-y_{2}\right)^{2} \\
-\left(x_{1}-x_{2}\right)\left(y_{1}-y_{2}\right) & \left(x_{1}-x_{2}\right)\left(y_{1}-y_{2}\right) & -\left(y_{1}-y_{2}\right)^{2} & \left(y_{1}-y_{2}\right)^{2}
\end{array}\right) \\
& \mathbf{K}_{G, G \text { Gatat }}=\frac{N}{L^{3}}\left(\begin{array}{cccc}
\left(y_{1}-y_{2}\right)^{2} & -\left(y_{1}-y_{2}\right)^{2} & -\left(x_{1}-x_{2}\right)\left(y_{1}-y_{2}\right) & \left(x_{1}-x_{2}\right)\left(y_{1}-y_{2}\right) \\
-\left(y_{1}-y_{2}\right)^{2} & \left(y_{1}-y_{2}\right)^{2} & \left(x_{1}-x_{2}\right)\left(y_{1}-y_{2}\right) & -\left(x_{1}-x_{2}\right)\left(y_{1}-y_{2}\right) \\
-\left(x_{1}-x_{2}\right)\left(y_{1}-y_{2}\right) & \left(x_{1}-x_{2}\right)\left(y_{1}-y_{2}\right) & \left(x_{1}-x_{2}\right)^{2} & -\left(x_{1}-x_{2}\right)^{2} \\
\left(x_{1}-x_{2}\right)\left(y_{1}-y_{2}\right) & -\left(x_{1}-x_{2}\right)\left(y_{1}-y_{2}\right) & -\left(x_{1}-x_{2}\right)^{2} & \left(x_{1}-x_{2}\right)^{2}
\end{array}\right)
\end{aligned}
$$

therefore, both stiffness matrices in Eq. (3) can be re-written as function of the Cartesian co-
The elastic stiffness matrix is based on the undeformed configuration of the structure (which does not depend on the loading conditions of the structure) whereas KG, the geometric stiffness matrix, accounts for the geometrical second order effects and depends on the axial force in the element.

The tangent stiffness matrix of the structure, KT, is given by: $\mathbf{K T}=\mathbf{K E}+\mathbf{K G}$.
In the context of tensegrities structures, authors [2-3] stated that the linear stiffness matrix, KE, and the geometrical stiffness matrix, KGhave been given respectively, by:

$$
\begin{aligned}
& \mathbf{K}_{E}=\mathbf{A} \operatorname{diag}\left(\frac{E \Omega}{L}\right) \mathbf{A}^{T} \\
& \mathbf{K}_{G}=\mathbf{I}_{d} \otimes \mathbf{D}
\end{aligned}
$$


Veenendaal and Block in [4] also proposed Eq. (7) for the geometrical stiffness matrix, KG, but for the lineal stiffness matrix, KE, they proposed the expression:

$$
\mathbf{K}_{E}=\mathbf{I}_{d} \otimes\left(\mathbf{C}^{\mathrm{T}} \operatorname{diag}(\mathbf{C} \mathbf{x})^{2} L^{-3} E \Omega \cdot \mathbf{C}\right)
$$

In the former equations represents the tensor product and $\mathrm{A}$ is the equilibrium matrix, defined as:

$$
\mathbf{A}=\left(\begin{array}{l}
\mathbf{C}^{\mathrm{T}} \operatorname{diag}(\mathbf{C x}) \\
\mathbf{C}^{\mathrm{T}} \operatorname{diag}(\mathbf{C y}) \\
\mathbf{C}^{\mathrm{T}} \operatorname{diag}(\mathbf{C z})
\end{array}\right)
$$

with $\mathrm{C}$ as the connectivity matrix (which is defined below) and $x, y$ and $z$ the node coordinate vectors in $\mathrm{x}, \mathrm{y}$ and $\mathrm{z}$ directions respectively.

$E$ is the modulus of Young

$\Omega$ is the area of the cross-section of each member

$L$ is the length of each pre-stressed member Id is the unit matrix in $\mathrm{Rd}$ ( $\mathrm{d}$ is the dimension of the structure: 2 for planar structures and 3 for 3D structures)

$D$ is the force density (or force/length coefficient) matrix, defined as:

$$
\mathbf{D}=\mathbf{C}^{\mathrm{T}} \operatorname{diag}(\mathbf{q}) \mathbf{C}
$$

with:

$$
\mathbf{q}=\left\{q_{1}, q_{2}, \ldots, q_{b}\right\}^{\mathrm{T}}=\left\{\frac{N_{1}}{L_{1}}, \frac{N_{2}}{L_{2}}, \ldots, \frac{N_{b}}{L_{b}}\right\}^{\mathrm{T}}
$$

The $\mathrm{i}$-component of vector $\mathrm{q}$ is the axial force to length ratio of member i (i.e. qi=Ni/Li). b is the number of branches or members of the tensegrity structure.

The connectivity or branch-node matrix $\mathrm{C}$,as discussed in[5], defines the connectivity of the nodes. It has one row per member in such a manner that each branch or connection $j$ links two nodesi and k, written asi(j) and $k(j)$ and are ordered so that $\mathrm{i}<\mathrm{k}$, indicating that they are part of the branch $j$, and the $i$ and $k$ elements of the $j$ row of $\mathrm{C}$ are set to 1 and -1 respectively, as follows:

$$
\mathbf{C}(j, r)=\left\{\begin{array}{c}
+1 \text { if } i(j)=r \\
-1 \text { if } k(j)=r \\
0 \text { for the rest }
\end{array}\right.
$$

The linear stiffness and geometric stiffness matrices of the 2D truss member shown in Figure 1 obtained from Eq. (6), (7) and (8) are, respectively:

$$
\mathbf{K}_{z}=\frac{E \Omega}{L}\left(\begin{array}{cccc}
\left(x_{1}-x_{2}\right)^{2} & -\left(x_{1}-x_{2}\right)^{2} & \left(x_{1}-x_{2}\right)\left(y_{1}-y_{2}\right) & -\left(x_{1}-x_{2}\right)\left(y_{1}-y_{2}\right) \\
-\left(x_{1}-x_{2}\right)^{2} & \left(x_{1}-x_{2}\right)^{2} & -\left(x_{1}-x_{2}\right)\left(y_{1}-y_{2}\right) & \left(x_{1}-x_{2}\right)\left(y_{1}-y_{2}\right) \\
\left(x_{1}-x_{2}\right)\left(y_{1}-y_{2}\right) & -\left(x_{1}-x_{2}\right)\left(y_{1}-y_{2}\right) & \left(y_{1}-y_{2}\right)^{2} & -\left(y_{1}-y_{2}\right)^{2} \\
-\left(x_{1}-x_{2}\right)\left(y_{1}-y_{2}\right) & \left(x_{1}-x_{2}\right)\left(y_{1}-y_{2}\right) & -\left(y_{1}-y_{2}\right)^{2} & \left(y_{1}-y_{2}\right)^{2}
\end{array}\right)
$$

$$
\begin{aligned}
& \mathbf{K}_{G}=\frac{N}{L}\left(\begin{array}{cccc}
1 & -1 & 0 & 0 \\
-1 & 1 & 0 & 0 \\
0 & 0 & 1 & -1 \\
0 & 0 & -1 & 1
\end{array}\right) \\
& \mathbf{K}_{E}=\frac{E \Omega}{L^{3}}\left(\begin{array}{cccc}
\left(x_{1}-x_{2}\right)^{2} & -\left(x_{1}-x_{2}\right)^{2} & 0 & 0 \\
-\left(x_{1}-x_{2}\right)^{2} & \left(x_{1}-x_{2}\right)^{2} & 0 & 0 \\
0 & 0 & \left(y_{1}-y_{2}\right)^{2} & -\left(y_{1}-y_{2}\right)^{2} \\
0 & 0 & -\left(y_{1}-y_{2}\right)^{2} & \left(y_{1}-y_{2}\right)^{2}
\end{array}\right)
\end{aligned}
$$


It is obvious that neither Eq. (13a) nor Eq. (13c) represent the linear stiffness matrix of the element. In the case of Eq. (13a) it is evident from a simple dimensional analysis. From the interpretation of the stiffness coefficients as displacement influence coefficients, Eq. (13c) cannot represent the linear stiffness matrix because it leads to uncoupled nodal forces and displacements, that is, a nodal $x$-force would only arise if a nodal $\mathrm{X}$-displacement is prescribed, which is not true.

In relation to the geometric stiffness matrix, KG, expression given by Eq. (13b) can be obtained from the strain energy accumulated in an elastic element subjected to axial loading assuming that the final and initial positions are close enough, a linear deformation field within the element and considering the tensor of Green-Lagrange strain tensor. It can be proved that theKG matrix given by Eq. (13b) remains unchanged with respect to the change of axes. According to Nermec et al. [6], the geometric stiffness matrix in Eq. (13b) introduces an unrealistic axial stiffening and lead to convergence problems, inaccuracies and even singularities for extreme compression. So, in order to avoid the above mentioned problems, the geometric stiffness of truss elements given by Eq. (1) or Eq. (5b), in local and global axis respectively, is widely used in structures.

If the notation employed in the form-finding problem of tension structures [5] is used, the classic linear stiffness and geometric stiffness matrices (i.e. Eq. (5a) and (5b), respectively) can be formulated as:

$$
\begin{aligned}
& \mathbf{K}_{E}=\mathbf{A} \operatorname{diag}\left(\frac{E \Omega}{L^{3}}\right) \mathbf{A}^{\mathrm{T}} \mathbf{K}_{G}=\mathbf{A}_{G} \operatorname{diag}\left(\frac{N}{L^{3}}\right) \mathbf{A}_{G}^{T} \\
& \text { with } \mathbf{A}_{G}=\left(\begin{array}{r}
-\mathbf{C}^{\mathrm{T}} \operatorname{diag}(\mathbf{C} \mathbf{y}) \\
\mathbf{C}^{\mathrm{T}} \operatorname{diag}(\mathbf{C} \mathbf{x})
\end{array}\right)
\end{aligned}
$$

At first sight, none of the stiffness matrices presented here (Eq.6, Eq.8 and Eq.14a) coincide. Obviously, the geometric stiffness matrices (Eq.7 and Eq.14b) do not coincide. It is clear that this point needs both clarification and homogeneity for future research and applicability.

\section{EXAMPLE}

In order to comment on both the influence and the validity of the foregoing expression of the stiffness matrix, the 2D tensegrity represented in Fig. 2, comprising 9 members and 6 nodes, has been analyzed. This structure is one of the most studied. Among other researchers' work, we can find it in $[02,03,07]$. The members are pin-connected at the perimeter, and do not intersect at the center.

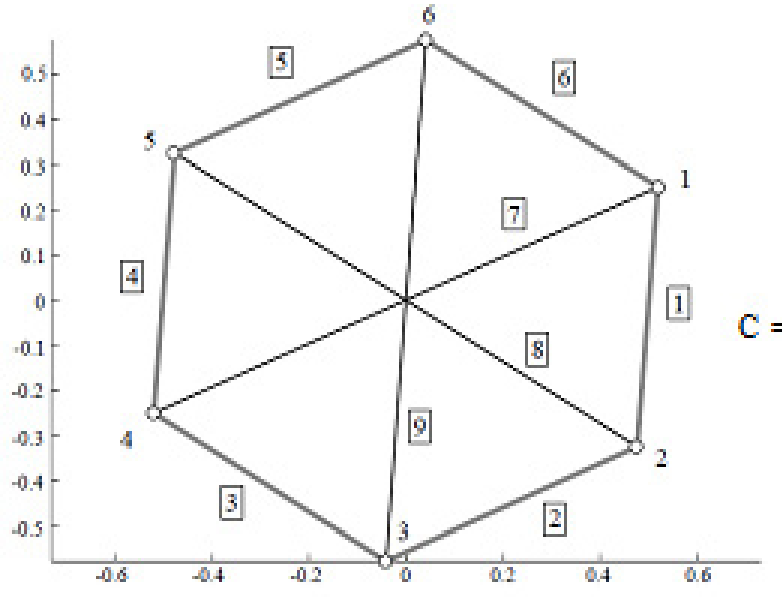

(a)

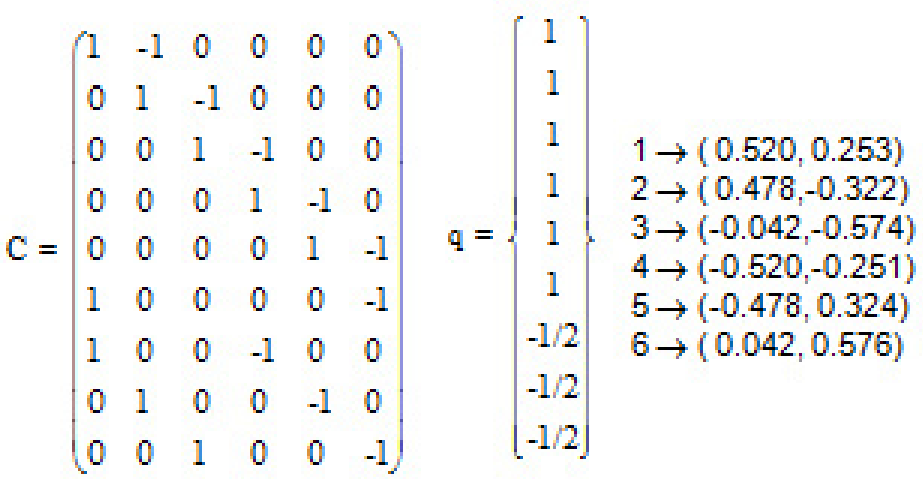

(b) (c) (d)

Figure 2: a) Equilibrium configuration of the $2 D$ tensegrity (wider gray lines (1 to 6) represent struts while thin black lines (7 to 8) represent cables); b) connectivity matrix; c) axial force to length ratios; d) coordinates of the points. Unit: $N$ and $m$. 
It is known that for a given structure, the momentat which buckling occurs (condition of elastic instability) may be expressed as a linear function of the displacements, as follows:

$$
\mathbf{K}_{E} \mathbf{d}-\mathbf{K}_{G} \mathbf{d}=\mathbf{0}
$$

where $\mathrm{KE}=$ the elastic structure stiffness matrix based on the undeformed configuration, $K G=$ the geometric stiffness matrix, which is defined in terms of a particular load pattern and accounts for second-order effects, and d= the vector containing the displacements at the degrees of freedom used in a discrete model of the structure. For a given load pattern, it is useful to express the geometric stiffness as a reference loading multiplied by a load factor ufor evaluating the critical buckling load of the structure:

$$
\mathbf{K}_{G}=\mu \cdot \mathbf{K}_{G 0}
$$

This is possible because the geometric stiffness matrix is proportional to the axial loads in the members of the structure. Consequently, in the following formulation, the loading applied to the structure is considered to be proportional to an initial loading pattern multiplied by the scalar $\mu$; that is, the relative distribution of the external loadsapplied to the structure is constant. A detailed discussion about this property of the geometric stiffness matrixas well as a development of Eq. (15) and (16)can be found in Clough and Penzien's text book [08].In this case, the initial pattern of KG0corresponds to the self-equilibratedaxial forces which pre-stress each branch in the equilibrium configuration, i.e, -see Figure 2-. The substitutionof Eq. (16) into Eq. (15) leads to thewell-known eigenvalue buckling problem:

$$
\left|\mathbf{K}_{E}-\mu \mathbf{K}_{G 0}\right|=\mathbf{0}
$$

A non-trivial solution from the lineal stability analysis - Eq. (17)-can be obtained if:

$$
\left|\mathbf{K}_{E}-\mu \mathbf{K}_{G 0}\right|=\mathbf{0}
$$

The smallest value of $\mu$ that satisfies Eq. (18)corresponds to the critical load multiplier of the structure [09].

In this example, the cross-sectional area of each branch and theelastic modulus are equal to 100 $\mathrm{mm} 2$ and $200000 \mathrm{MPa}$, respectively.

In Table 1 the obtained eigenvalues of the buckling problem corresponding to the tensegrity in
Figure 2 are summarized. In Table 1, the expression from which each matrix is obtained is indicated in the first two columns. The last row shows the critical load multiplier obtained using the ANSYS@ software, members were modeled with the LINK8 element type. The pre-stressed loads were introduced through initial strains to simulate tension or compression.

From comparison of the two last rows in Table 1 , it is evident that the result obtained from $\mathrm{FE}$ analysis coincides with the theoretical one obtained from the proposed matrices Eq. (14 a. and $b$ ).

\begin{tabular}{|c|c|c|}
\hline \multicolumn{2}{|c|}{ Buckling eigenvalue: } & $K E-\mu K G 0 \mid=0$ \\
\hline KE & KG0 & $\mu$ \\
\hline Eq. (6) & Eq. (7) & $2.50 \cdot 10^{7}$ \\
\hline Eq. (8) & Eq. (7) & $2.79 \cdot 10^{7}$ \\
\hline Eq. (14a) & Eq. (14b) & $2.21 \cdot 10^{7}$ \\
\hline \multicolumn{2}{|c|}{ FE analysis with ANSYS } & $2.22 \cdot 10^{7}$ \\
\hline
\end{tabular}

Table 1: Summary of eigenvalues of the buckling problem obtained considering different matrices and using finite element analysis

\section{CONCLUSION}

In the context of tensegrity structures, the stiffness matrix can be obtained from the two matrices used in the shape-finding problem of pinjointed structures (matrix A given in Eq. (9)) and the force to length ratios (matrix $D$ given in $E q$. (10)). Expressions for both, linear and geometric, stiffness matrices have been presented.

\section{REFERENCES}

1) O. C. Zienkiewicz, The finite element method. 3rdEd. McGraw-Hill (London). (1977).

2) G.G. Estrada, H.J. Bungartz, C. Mohrdieck, Numerical form-finding of tensegrity structures. International Journal of Solid and structures 43 (2006) 6855-6868

3) H.C. Tran, J. Lee, Advanced form-finding of tensegrity structures. Computers and Structures 88 (2010) 237-246.

4) D. Veenendaal, P. Block, An overview and comparison of structural form finding methods for general networks. International Journal of Solids and structures 49 (2012) 37413753.

5) H. J. Schek, The force density method for form-finding and computation of general 
networks. Computer Methods in Applied Mechanics and Engineering, vol. 3, (1974) 115-134.

6) I. Němec, M. Trcala, I. Ševčík, H. Štekbauer, New Formula for Geometric Stiffness Matrix Calculation. Journal of Applied Mathematics and Physics, 4, (2016) 733-748.

7) A. G. Tibert, S. Pellegrino, Review of FormFinding Methods for Tensegrity Structures. International Journal of Space Structures, vol. 18, no. 4, (2009) 209-223.
8) R. W. Clough, J. Penzien, Dynamics of structures. 2nd ed. New York: McGraw-Hill, Inc. (1993).

9) L.M.Gil-Martín, E. Hernández-Montes, M. Aschheim, Optimal design of planar frames based on stability criterion using first-order analysis, Engineering Structures 28, (2006) 1780-1786.

Paper sent to revision: 15.02.2016.

Paper ready for publication: 15.09.2017. 